\title{
Mitochondria-Targeted Antioxidant Mito-Tempo Protects Against Aldosterone-Induced Renal Injury In Vivo
}

\author{
Wei Ding ${ }^{a} \quad$ Tingyan Liu $^{\mathrm{a}} \quad \mathrm{Xiao}^{\mathrm{B}} \mathrm{Bi}^{\mathrm{a}} \quad$ Zhiling Zhang ${ }^{\mathrm{b}}$ \\ aDivision of Nephrology, Shanghai Ninth People's Hospital, School of Medicine, Shanghai Jiaotong \\ University, Shanghai, bEmergency Department, Shanghai Ninth People's Hospital, School of Medicine, \\ Shanghai Jiaotong University, Shanghai, China
}

\section{Key Words}

Aldosterone - Mitochondrial dysfunction - Nlrp3 inflammasome - Endoplasmic reticulum stress $\cdot$ Mito-Tempo

\begin{abstract}
Background/Aims: Growing evidence suggests mitochondrial dysfunction (MtD) and the Nlrp3 inflammasome play critical roles in chronic kidney disease (CKD) progression. We previously reported that Aldosterone (Aldo)-induced renal injury in vitro is directly caused by mitochondrial reactive oxygen species (mtROS)-mediated activation of the Nlrp3 inflammasome. Here we aimed to determine whether a mitochondria-targeted antioxidant (Mito-Tempo) could prevent Aldo-induced kidney damage in vivo. Methods: C57BL/6J mice were treated with Aldo and/or Mito-Tempo (or ethanol as a control) for 4 weeks. Renal injury was evaluated by Periodic Acid-Schiff reagent or Masson's trichrome staining and electron microscopy. ROS were measured by DCFDA fluorescence and ELISA. MtD was determined by real-time PCR and electron microscopy. Activation of the Nlrp3 inflammasome and endoplasmic reticulum stress (ERS) was detected via western blot. Results: Compared with control mice, Aldo-infused mice showed impaired renal function, increased mtROS production and MtD, Nlrp3 inflammasome activation, and elevated ERS. We showed administration of Mito-Tempo significantly improved renal function and MtD, and reduced Nlrp3 inflammasome activation and ERS in vivo. Conclusion: Mitochondria-targeted antioxidants may attenuate Aldo-infused renal injury by inhibiting MtD, the NIrp3 inflammasome, and ERS in vivo. Therefore, targeting mtROS might be an effective strategy for preventing CKD.
\end{abstract}

(C) 2017 The Author(s)

Published by S. Karger AG, Basel

\section{Introduction}

Aldosterone (Aldo) is a key regulator of electrolyte balance in the body: it acts classically via the mineralocorticoid receptor (MR) to stimulate sodium reabsorption, leading to W. Ding and T. Liu contributed equally to this work. 
increased blood pressure. In addition to hypertension, growing evidence indicates chronic administration of Aldo causes glomerulosclerosis and renal fibrosis [1]. In addition, clinical research has shown that MR antagonists decrease albumin excretion in diabetic patients, and reduce the risk of morbidity and mortality in patients with heart failure [2, 3]. Furthermore, Epstein and colleagues reported that in patients with hyperaldosteronism exhibiting the "Aldo escape phenomenon", a low dose MR antagonist had a protective effect on the target organs [4]. Animal studies also indicate that Aldo directly provokes renal injury through inducing oxidative stress and producing inflammatory and pro-fibrotic cytokines [5]. Therefore, inhibiting Aldo-mediated effects may help prevent renal damage and the development of chronic kidney disease (CKD).

The underlying mechanism of Aldo-induced renal injury has been increasingly investigated. Aldo has been shown to induce the production of reactive oxygen species (ROS) in kidney cells (including hydrogen peroxide, superoxide, and hydroxyl radicals), which eventually results in renal injury. The mitochondrial respiratory chain is a primary source of ROS: unpaired electrons escape the transport chain and react with oxygen to form ROS [6]. Overproduction of ROS damages the oxidative respiratory chain and mitochondrial DNA (mtDNA), ultimately leading to mitochondrial dysfunction (MtD) [7].

MtD is characterized by increased ROS generation, progressive respiratory chain dysfunction, and accumulation of impaired mtDNA [8]. We previously demonstrated that mitochondrial ROS (mtROS) contribute to Aldo-induced renal tubular injury in vitro. We also showed that rotenone, an inhibitor of mitochondrial complex I, attenuated Aldo-induced renal injury by inhibiting oxidative stress and mitochondrial dysfunction [9, 10]. These results suggest MtD is an important factor in Aldo-induced kidney disease.

There is also increasing evidence that an increased inflammasome-mediated inflammatory response is involved in cellular dysfunction during CKD. Among the inflammasomes identified to date, the Nucleotide-binding oligomerization domain [NOD]like pyrin domain containing protein 3 (Nlrp3) inflammasome is the best characterized [11]. The Nlrp3 inflammasome is a multiprotein complex expressed in the cytosol of immune cells that is activated in response to various triggers including danger signals, such as mtROS, extracellular ATP, and damaged nucleic acids [12]. Activation of the Nlrp3 inflammasome leads to autocatalytic activation of caspase-1, which then cleaves pro-IL-1 $\beta$ and pro-IL-18 cytokines into their mature forms. We previously showed that mtROS-mediated activation of the Nlrp3 inflammasome directly caused renal tubular epithelial injury. We also found that treatment with a mitochondria-targeted antioxidant (Mito-Tempo or MT) significantly attenuated Aldo-induced apoptosis of human kidney cells (HK-2 cells) and the activation of Nlrp3 inflammasome in vitro.

MT combines the antioxidant piperidine nitroxide (Tempo) with the lipophilic cation triphenylphosphonium ( $\mathrm{TPP}^{+}$) for mitochondria targeting [13]. Along with its beneficial results on kidney cells in vitro [9], MT was recently shown to decrease tumor necrosis factor- $\alpha$ (TNF- $\alpha$ )-induced mtROS and apoptosis in H9c2 cells, inhibit mitochondrial superoxide levels, and attenuate survival in a murine model of sepsis $[14,15]$. Based on the abovementioned results, in this study we aimed to investigate whether MT could prevent Aldo-induced renal injury in vivo.

\section{Materials and Methods}

Reagents and Antibodies

Aldo, 2',7'-dichlorofluorescein diacetate, and MT were purchased from Sigma (St. Louis, MO). Mouse monoclonal anti-Nlrp3 antibody and rabbit polyclonal anti-ASC antibody were purchased from adipoGen (San Diego, CA). Rabbit polyclonal anti-IL-18 and anti-caspase-12 were purchased from Santa Cruz Biotechnology (Santa Cruz, CA). Rabbit polyclonal anti-GRP78, anti-GRP94, and anti-CHOP were purchased from Cell Signaling Technology. Goat polyclonal anti-IL-1 $\beta$ was purchased from R\&D systems (Minneapolis, $\mathrm{MN})$.

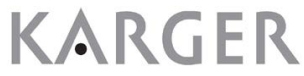




\section{Cellular Physiology Cell Physiol Biochem 2017;44:741-750

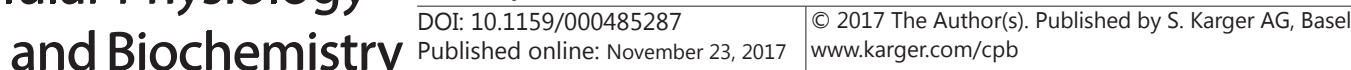

Ding et al.: Mitochondrial Reactive Oxygen Species \& Kidney Disease

Animal Preparation

All experimental procedures were performed according to our previous studies. The experiments were performed according to the Shanghai Jiaotong University's guide for laboratory animals. In brief, 8-week old C57BL/6J mice (male, 20-25g) were obtained from the Shanghai SLAC Lab and underwent right uninephrectomy under anesthesia with sodium pentobarbital (50 mg/kg, i.p.). All mice were given drinking water containing $1 \% \mathrm{NaCl}$ after 2 weeks of recovery. Mice were divided into four groups and treated for 4 weeks as follows: Sham group (0.5\% ethanol subcutaneously, saline vehicle i.p., $n=6$ ); Aldo group (Aldo 0.75 $\mu \mathrm{g} / \mathrm{h}$ subcutaneously, saline vehicle i.p., $n=7$ ); Aldo+MT group (Aldo $0.75 \mu \mathrm{g} / \mathrm{h}$ subcutaneously, $1 \mathrm{mg} / \mathrm{kg}$ / day MT i.p., $n=7)$; MT group (0.5\% ethanol subcutaneously, $1 \mathrm{mg} / \mathrm{kg} /$ day MT i.p., $n=7)$. An osmotic minipump was implanted subcutaneously to infuse either ethanol or Aldo. At the end of the experiment, the mice were anesthetized and kidney sections were fixed in 10\% formalin and embedded in paraffin. In addition, the plasma was centrifuged for measuring IL-1 $\beta$ and IL-18 via ELISA (ELISA kits; RayBiotech, Norcross, GA). Kidney samples were immediately frozen in liquid nitrogen and stored at $-80^{\circ} \mathrm{C}$ [16].

\section{Histological Examination}

The kidneys were sectioned (thickness $3 \mu \mathrm{m}$ ), processed, and stained with Periodic Acid-Schiff reagent (PAS) or Masson's trichrome stain as per our previous study. After PAS staining, the severity of glomerular injury was evaluated in experimental mice using light microscopy in accordance with the following semiquantitative grades: grade 0 , normal; grade 1 , segmental lesion $<25 \%$; grade $2,25 \%-50 \%$; grade $3,50 \%-$ $75 \%$; grade 4,75\%-100\%. At least 20 glomeruli were analyzed in each group. The area of tubulointerstitial fibrosis was also measured in 10 random 400x magnification fields following Masson's trichrome staining [17].

\section{Electron Microscopy Examination}

To detect podocyte foot processes and mitochondrial ultrastructure morphology, we cut renal sample into $1 \mathrm{~mm}^{3}$ pieces using a scalpel. We cut ultrathin sections on a microtome, placed them on copper grids, stained them with uranyl acetate and lead citrate, and visualized them with an electron microscope. All these experiments were performed as described previously [10].

\section{Detection of ROS}

Renal ROS generation was detected using 2',7'-dichlorofluorescein diacetate (DCFDA) fluorescence. Renal ROS levels were measured in a $4 \mu \mathrm{m}$ tissue cryosection, which was treated with $10 \mu \mathrm{M}$ DCFDA in the dark at $37^{\circ} \mathrm{C}$ for $30 \mathrm{~min}$. At the end of the process, the slides were washed using PBS and imaged by fluorescence microscopy [18]. Malondialdehyde (MDA) levels were detected with commercial kits (Jiancheng Research Institute, China), and 8-OHdG levels were determined using an ELISA kit (BMAssay, China) according to the manufacturer's instructions.

\section{Real-Time PCR}

Total RNA was isolated from the renal cortex, reverse transcribed to cDNA, and subjected to PCR amplification, according to our previous study. The primers were as follows: ATP synthase: forward 5'-TCCATCAAAAACATCCAGAAAA-3' and reverse 5'-GAGGAGTGAATAGCACCACAAA-3'; 18S: forward 5'-TTCGGAACTGAGGCCATGATT-3' and reverse 5'-TTTCGCTCTGGTCCGTCTTG-3'; mtDNA: forward 5'-TTTTATCTGCATCTGAGTTTAATCCTGT-3' and reverse 5'-CCACTTCATCTTACCATTTATTATCGC-3' [16].

\section{Western Blot Analysis}

Renal protein was denatured in boiling water for $10 \mathrm{~min}$, then separated by SDS-PAGE gel and transferred to nitrocellulose membranes. The blots were blocked by $5 \%$ non-fat milk, followed by incubation for $2 \mathrm{~h}$ with anti-Nlrp3, anti-ASC, anti-IL-1 $\beta$, anti-IL-18, anti-GRP78, anti-GRP94, anti-CHOP, and anti-Caspase-12 antibodies, all at a dilution of 1:1000. After washing and incubating with horseradish peroxidase (HRP)conjugated secondary antibodies, the blots were visualized with enhanced chemiluminescence (ECL) kits.

Statistical Analysis

All data is presented as means \pm SEM. One-way ANOVA for repeated measures was used to compare mean values between two groups. A $P<0.05$ was considered statistically significant. 


\section{Cellular Physiology

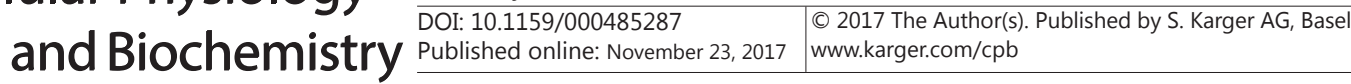

Ding et al.: Mitochondrial Reactive Oxygen Species \& Kidney Disease

\section{Results}

\section{Effects of MT on renal histology}

Mesangial matrix expansion was evident in the glomeruli of the Aldo-infused group. Compared with the control (Sham) group, PAS-positive mesangial matrix areas were significantly larger in Aldo-infused mice (Fig. 1A). However, treatment with MT significantly attenuated renal injury and decreased glomerular injury score (Figs. 1A, 2B). Similarly, quantitative determination of the tubulointerstitial fibrosis index revealed that Aldo markedly induced renal fibrosis compared with the Sham group; however, the Aldo-induced renal fibrosis was inhibited by MT treatment (Figs. 1B, 2C). Aldo-infused mice also showed

Fig. 1. MT attenuates renal fibrosis in Aldo-infused mice. Representative photomicrographs of Periodic Acid-Schiff reagent (PAS) stained kidney sections (A, magnification, $\times 400$ ) and Masson's trichrome stained kidney sections (B, magnification, $\times 400$ ).
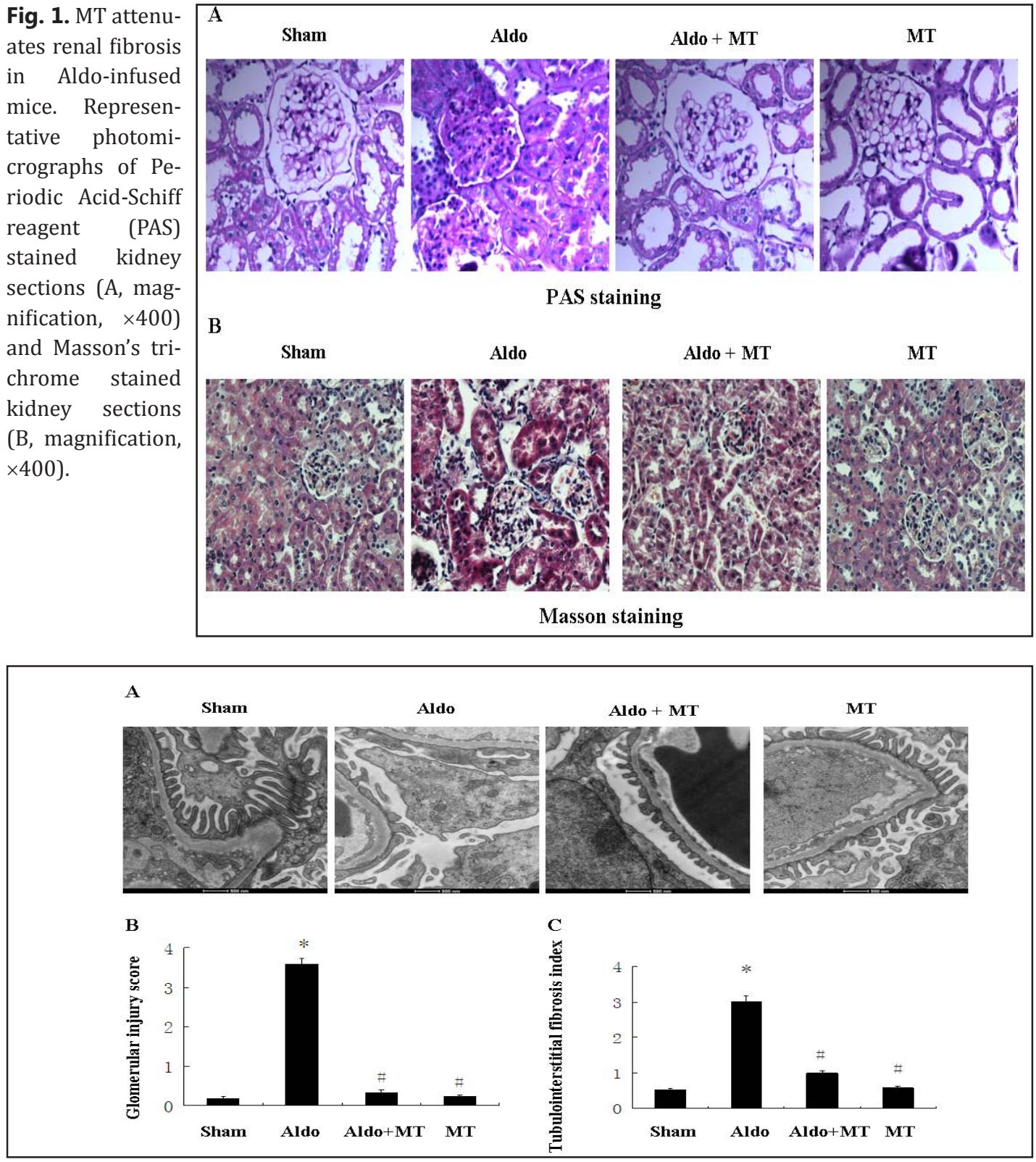

Fig. 2. MT attenuates Aldo-induced kidney damage. (A) Representative electron microscopy photomicrographs of morphology changes of podocyte foot processes in mice from the four groups (magnification $\times 12,000$ ). (B) Glomerular injury score and (C) tubulointerstitial fibrosis index in mice from the four groups. All values are means $\pm \operatorname{SEM}(n=6) .{ }^{*} \mathrm{P}<0.05$ versus Sham group; ${ }^{~} \mathrm{P}<0.05$ versus Aldo group. 


\section{Cellular Physiology

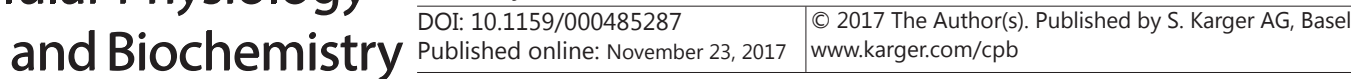

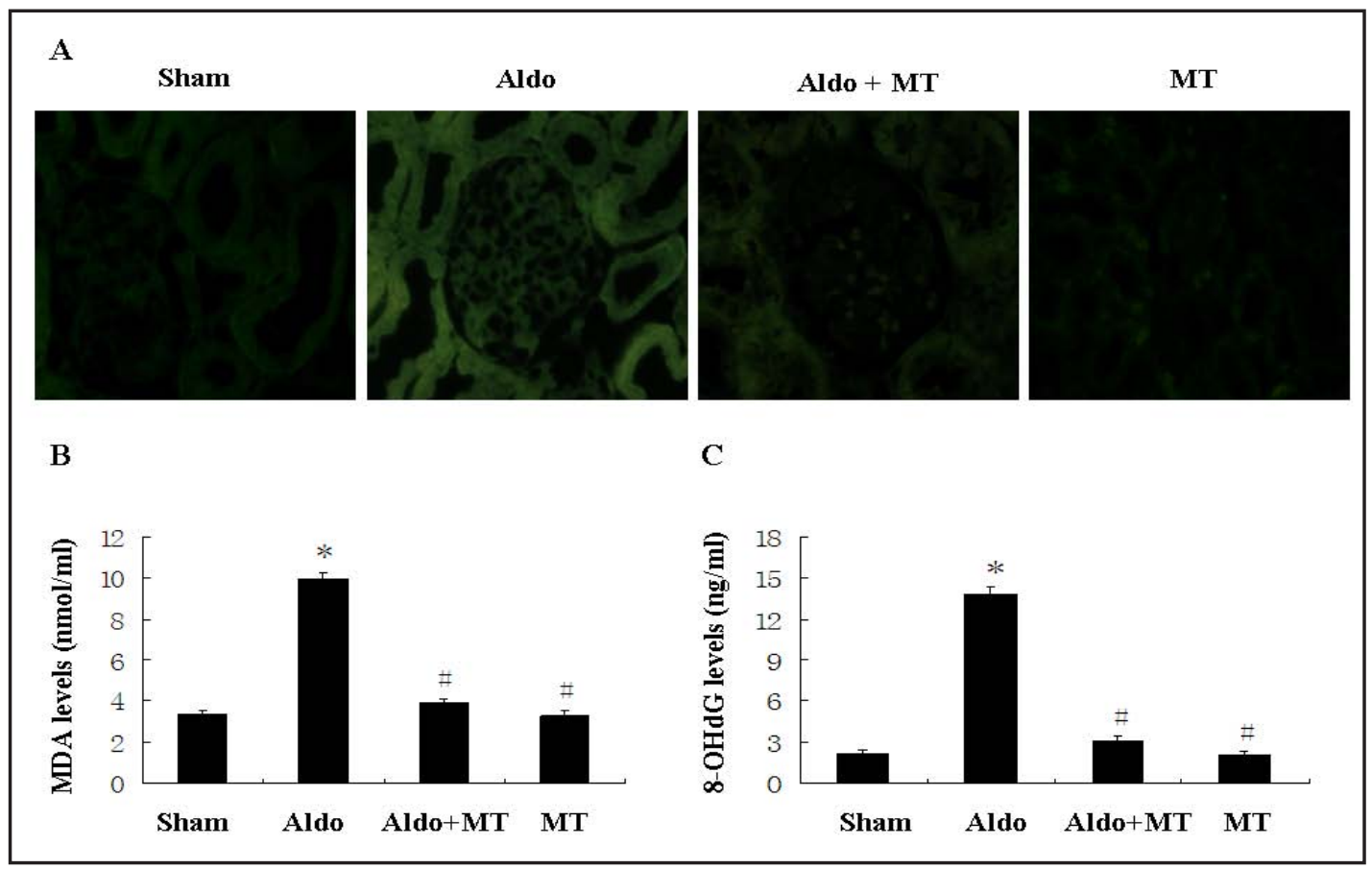

Fig. 3. MT attenuates Aldo-induced ROS production. (A) Representative images of DCFDA fluorescence in kidney sections from mice in the four groups (magnification, $\times 400$ ). (B) Serum MDA and (C) 8-OHdG levels in mice from the four groups. All values are means \pm SEM $(n=6) .{ }^{*} \mathrm{P}<0.05$ versus Sham group; ${ }^{\mathrm{P}}<0.05$ versus Aldo group.

disappearance of the slit diaphragm gap and foot process effacement compared with the Sham group, while treatment with MT markedly ameliorated these changes in renal ultrastructure morphology (Fig. 2A).

\section{Effects of MT on renal ROS}

Aldo-treated mice exhibited increased production of renal ROS: DCFDA fluorescence was increased, and serum levels of MDA and 8-OHdGs (markers of oxidative stress) were elevated (Fig. 3). Conversely, a notable decrease in serum MDA and 8-OHdG levels was observed upon treatment with MT, along with decreased levels of renal ROS measured using DCFDA fluorescence (Fig. 3).

\section{Effects of MT on mitochondrial dysfunction}

Following 4 weeks of Aldo-infused renal injury, we evaluated MtD via examination of mtDNA and ATP synthase levels using real-time PCR. As expected, Aldo-infusion resulted in a striking reduction of mtDNA and ATP synthase levels, indicative of mitochondrial abnormality (Fig. 4). However, treatment with MT significantly attenuated MtD (Fig. 4). In addition, renal cells from the Aldo-infused group showed swollen mitochondria with disorganized and fragmented cristae, while these effects were significantly ameliorated in MT treatment group (Fig. 4A).

\section{Effects of MT on Nlrp3 inflammasome activation}

According to our previous study, mitochondrial ROS-mediated Nlrp3 inflammasome activation contributes to Aldo-induced tubular injury in vitro. To investigate the role of MT on Nlrp3 inflammasome activation in Aldo-induced renal injury in vivo, we detected Nlrp3, ASC, IL-1 $\beta$, and IL-18 levels using western blot. As shown in Fig. 5, renal Nlrp3 and ASC protein levels were significantly upregulated in the Aldo-infused group, while treatment 


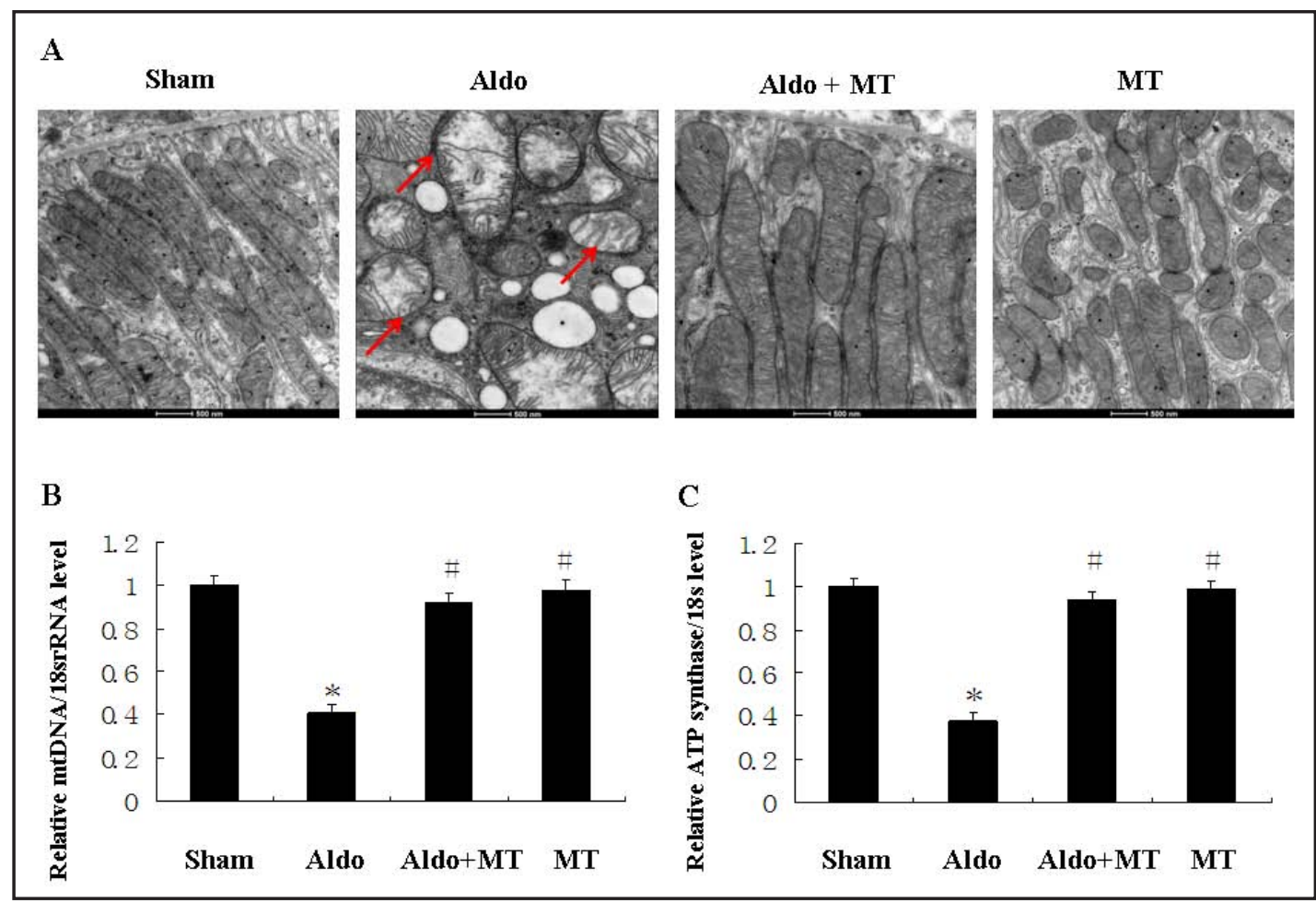

Fig. 4. MT attenuates Aldo-induced mitochondrial dysfunction. (A) Representative electron microscopy photomicrographs of ultrastructural morphology of mitochondria in mice from the four groups (magnification $\times 12,000$ ); red arrow indicates swollen mitochondria. (B) Semi-quantitative analysis of mitochondrial DNA (mtDNA) levels and (C) ATP synthase levels in mice from the four groups normalized against 18S performed by real-time PCR. All values are means \pm SEM $(n=6)$. ${ }^{*} \mathrm{P}<0.05$ versus Sham group; $\mathrm{P}<0.05$ versus Aldo group.

Fig. 5. MT attenuates Aldo-induced activation of the Nlrp3 inflammasome in vivo. (A) Expression of renal Nlrp3 protein via western blot and (B) levels normalized against -actin in mice from the four groups. (C) Expression of renal ASC protein via western blot and (D) levels normalized against $\beta$-actin in mice from the four groups. All values are means \pm SEM $(\mathrm{n}=$ 6). ${ }^{*} \mathrm{P}<0.05$ versus Sham group; $\quad{ }^{*} \mathrm{P}<0.05$ versus Aldo group.

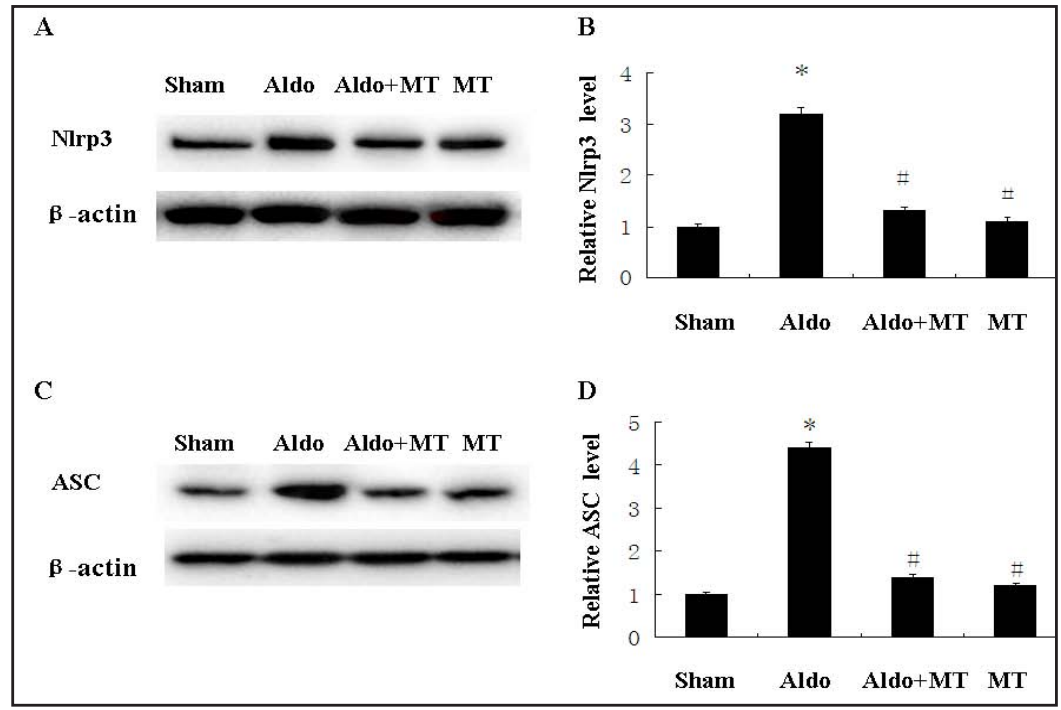

with MT markedly inhibited Nlrp3 inflammasome activation. Similarly, renal IL-1 $\beta$ and IL-18 protein levels were increased in the Aldo-infused group; these effects were accompanied by significantly increased serum IL-1 $\beta$ and IL-18 levels (Fig. 6). However, administration of MT decreased the levels of these inflammatory cytokines (Fig. 6). 
Fig. 6. MT attenuates Aldo-induced Nlrp3 inflammasome-regulated cytokines. (A) Expression of renal IL$1 \beta$ and IL-18 proteins via western blot and (B) levels normalized against -actin in mice from the four groups. Serum IL-1 $\beta$ (C) and IL18 (D) levels detected via ELISA. All values are means \pm SEM (n = 6). ${ }^{*} \mathrm{P}<0.05$ versus Sham group; ${ }^{*} \mathrm{P}<0.05$ versus Aldo group.

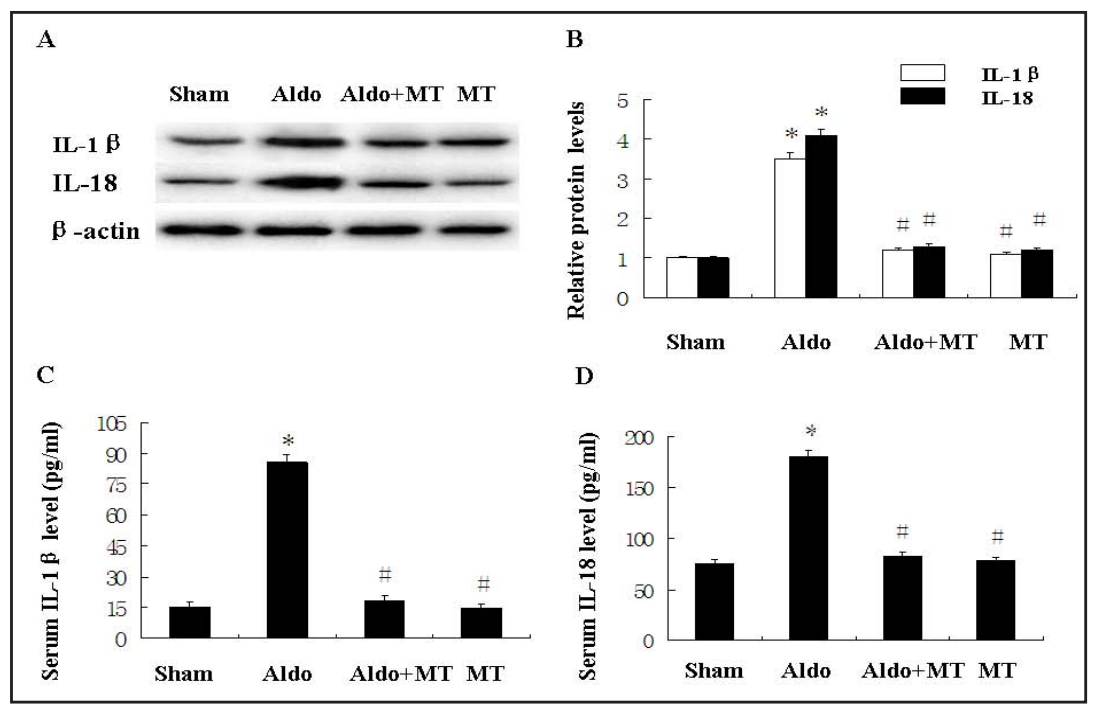

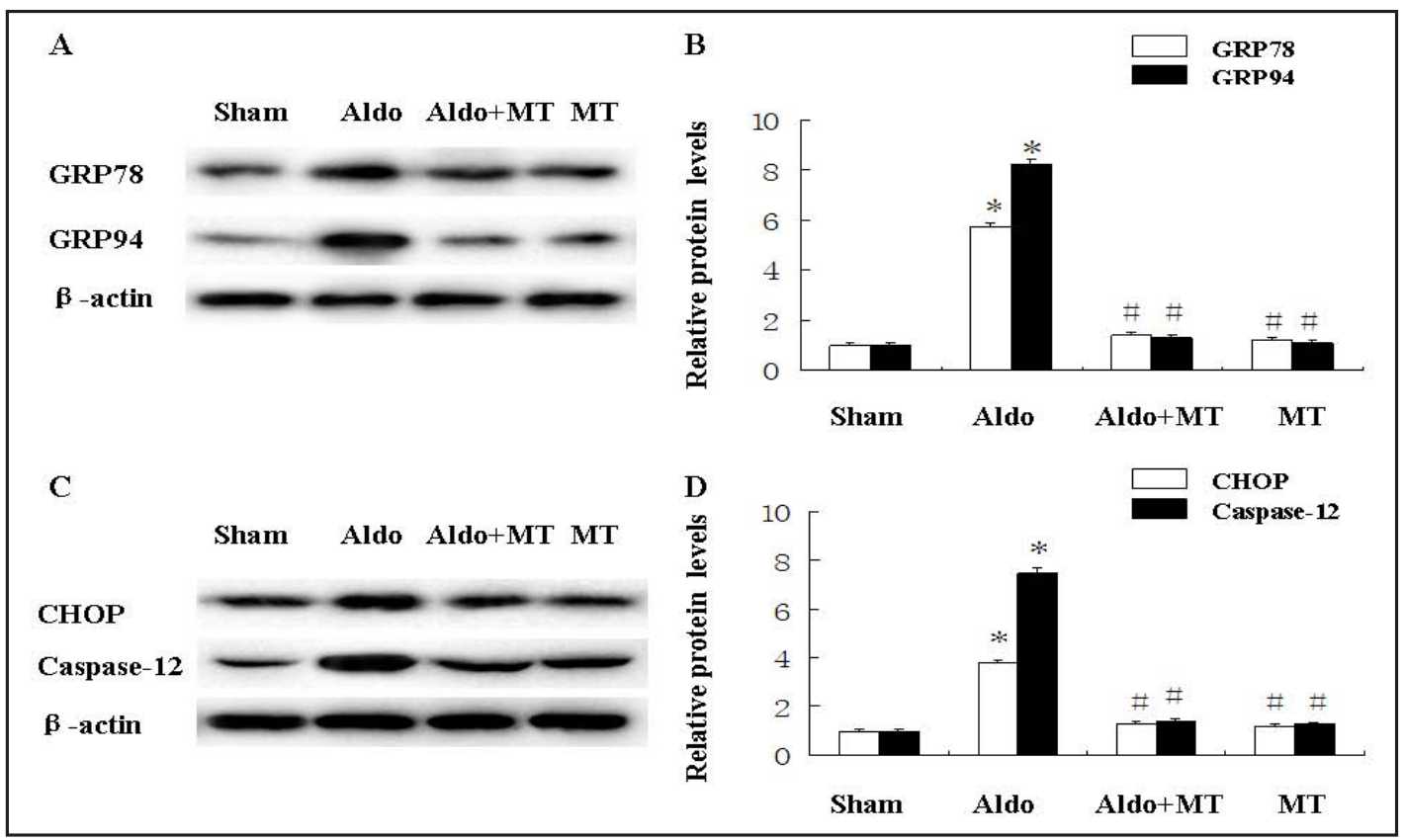

Fig. 7. MT attenuates Aldo-induced endoplasmic reticulum stress. (A) Expression of renal GRP78 and GRP94 proteins via western blot and (B) levels normalized against $\beta$-actin in mice from the four groups. (C) Expression of renal Chop and Caspase-12 proteins via western blot and (D) levels normalized against $\beta$-actin in mice from the four groups. All values are means $\pm \operatorname{SEM}(n=6) .{ }^{*} \mathrm{P}<0.05$ versus Sham group; ${ }^{\#} \mathrm{P}<0.05$ versus Aldo group.

\section{Effects of MT on endoplasmic reticulum stress}

Growing evidence suggests that endoplasmic reticulum stress (ERS) is involved in Aldo-induced renal injury $[18,19]$. Compared with the Sham group, Aldo-infused mice showed increased expression of the ERS-related proteins GRP78 and GRP94 (Fig. 7). Meanwhile, treatment with MT significantly decreased GRP78 and GRP94 levels (Fig. 7). The ERS-mediated apoptosis pathway was also affected; in particular, levels of the CHOP and caspase-12 proteins were markedly increased in the Aldo-infused group, and these changes were attenuated following treatment with MT (Fig. 7). 


\section{Discussion}

In the present in vivo study, the protective role of the mitochondria-targeted antioxidant Mito-Tempo in Aldo-induced renal injury was investigated. We showed that Aldo infusion significantly induced renal damage in mice, as characterized by an increased glomerular injury score, tubulointerstitial fibrosis index, and foot process effacement. Moreover, renal ROS generation and MtD increased in Aldo-infused mice, the Nlrp3 inflammasome was activated, and there was evidence of ERS. We also found that treatment with Mito-Tempo demonstrated beneficial effects on MtD, Nlrp3 inflammasome activation, ERS, and renal fibrosis. Therefore, mtROS may be a potential therapeutic target for reducing Aldo-induced kidney damage and renal fibrosis.

ROS are generated in response to various kinds of physiological and environmental stresses, and have been implicated in the pathogenesis of inflammatory diseases including thyroiditis, multiple sclerosis, and CKD [20, 21]. As ROS are primarily produced in mitochondria, MtD can increase ROS levels, thereby contributing to disease progression [22]. Indeed, MtD was recently shown to cause a wide range of kidney diseases; for example, two children with focal segmental glomerulosclerosis (FSGS) had mtDNA mutations in podocytes [23] and MtD was shown to be an early event in Aldo-induced podocyte injury [24]. As further evidence, our previous study showed MtD mediated renal fibrosis in a mouse unilateral ureteral obstruction (UUO) model of CKD [17]. In the present study, we confirmed this phenomenon: Aldo-infused mice showed reduced mtDNA and ATP synthase levels, and their mitochondria were swollen with disorganized and fragmented cristae. Furthermore, pretreatment with the antioxidant Mito-Tempo not only decreased accumulation of ROS and $\mathrm{MtD}$, but also attenuated renal injury in Aldo-infused mice.

Recent evidence also suggests that the Nlrp3 inflammasome plays a key role in the pathogenesis of renal disease. Homsi et al. reported that treatment with a caspase-1 inhibitor reduced inflammatory responses and attenuated renal function in a rat model of RIAKI [25]. In addition, Vilaysane et al. showed that activation of the Nlrp3 inflammasome is involved in UUO-induced renal inflammation and leads to renal fibrosis [26]. Recently, it was also demonstrated that an Nlrp3-specific inflammasome inhibitor significantly improved crystal-induced renal inflammation and fibrosis [27]. We previously showed that mtROSmediated Nlrp3 inflammasome activation directly contributed to Aldo-induced renal injury in vitro. In the present study, we found Mito-Tempo treatment ameliorated renal injury by decreasing mtROS, improving MtD, and preventing activation of the Nlrp3 inflammasome in Aldo-infused mice. These studies suggest the mtROS/Nlrp3 inflammasome axis contributes to Aldo-induced renal injury, as confirmed by both in vitro and in vivo studies.

Previous studies have also indicated that ERS plays a critical role in the progression of CKD. The ER is responsible for protein synthesis, folding, and maturation, and participates in $\mathrm{Ca}^{2+}$ storage, signal transduction, lipid, and glucose metabolism. Abnormalities in these factors could cause unfolding or misfolding of proteins, which accumulate in the ER lumen leading to ERS and the unfolded protein response [28, 29]. ERS has been shown to contribute to Aldo-induced renal injury, while treatment with ERS inhibitors (e.g. chemical ER chaperones like tauroursodeoxycholic acid) attenuate Aldo-induced renal injury and uremic cardiomyopathy $[16,30]$.

Mitochondrial oxidative stress and ERS are closely entwined pathological states that usually occur simultaneously. ROS are thought to act as messengers between the mitochondria and the ER. Oxidative stress and ERS are interconnected in that protein misfolding in the ER leads to ROS accumulation and in turn, oxidative stress could disturb the ER redox state, leading to increased protein misfolding [31]. Indeed, in the present study, we demonstrated increased ROS, MtD, and activation of the ERS-related apoptosis pathway were involved in Aldo-induced kidney damage in mice. We also found that treatment with the mitochondriatargeted antioxidant Mito-Tempo significantly reduced levels of ERS proteins (GRP78 and GRP94) and inhibited activation of the ERS apoptotic pathway. These results suggest MitoTempo may attenuate renal injury by targeting mitochondria-ER interplay in CKD.

\section{KARGER}




\section{Cellular Physiology Cell Physiol Biochem 2017;44:741-750 \begin{tabular}{l|l} 
and Biochemistry Published online: November 23, 2017 & $\begin{array}{l}\text { (c) } 2017 \text { The Author(s). Published by S. Karger AG, Basel } \\
\text { www.karger.com/cpb }\end{array}$
\end{tabular} \\ Ding et al.: Mitochondrial Reactive Oxygen Species \& Kidney Disease}

Treatment with the mitochondria-targeted antioxidant Mito-Tempo was previously shown to significantly decrease mtROS generation and mtDNA translocation in response to LPS and ATP $[13,32]$. In the present study, we evaluated the role of Mito-Tempo in antagonizing Aldo-induced renal injury in mice. Consistent with our previous in vitro results [9], Aldo infusion significantly induced MtD, Nlrp3 inflammasome activation, ERS, and renal damage. Meanwhile, mice treated with Mito-Tempo showed marked improvement in renal injury, as evidenced by decreased mtROS levels, Nlrp3 inflammasome activation, and ERS activation. Together, our data suggests that Mito-Tempo could protect against Aldo-induced renal injury in vivo.

\section{Conclusion}

Our results indicate Mito-Tempo plays an important role in attenuating Aldo-infused renal injury in vivo, possibly by inhibiting mtROS production and MtD, and preventing Nlrp3 inflammasome and ERS activation. Therefore, targeting mtROS might be an effective strategy in ameliorating Aldo-induced renal injury and disease progression in CKD.

\section{Acknowledgements}

This work was supported by grants from the Funds for distinguished Young Scholar of Ninth people's hospital (jyyq09201701) and National Natural Science Foundation of China (81300590).

\section{Disclosure statement}

The authors declare that there is no Disclosure Statement.

\section{References}

1 Funder JW: Aldosterone and mineralocorticoid receptors: orphan questions. Kidney Int 2000; 57:13581363.

2 Epstein M, Williams GH, Weinberger M, Lewin A, Krause S, Mukherjee R, Patni R, Beckerman B: Selective aldosterone blockade with eplerenone reduces albuminuria in patients with type 2 diabetes. Clin J Am Soc Nephrol 2006;1:940-951.

-3 Pitt B, Remme W, Zannad F, Neaton J, Martinez F, Roniker B, Bittman R, Hurley S, Kleiman J, Gatlin M: Eplerenone, a selective aldosterone blocker, in patients with left ventricular dysfunction after myocardial infarction. N Engl J Med 2003; 348:1309-1321.

4 Epstein M: Aldosterone blockade: an emerging strategy for abrogating progressive renal disease. Am J Med 2006; 119:912-919.

>5 Kiyomoto H, Rafiq K, Mostofa M, Nishiyama A:. Possible underlying mechanisms responsible for aldosterone and mineralocorticoid receptor-dependent renal injury. J Pharmacol Sci 2008;108: 399-405.

6 Wang Z, Cai F, Chen X, Luo M, Hu L, Lu Y: The role of mitochondria-derived reactive oxygen species in hyperthermia-induced platelet apoptosis. PLoS One 2013;8:e75044.

7 Yuan Y, Wang H, Wu Y, Zhang B, Wang N, Mao H, Xing C: P53 contributes to cisplatin induced renal oxidative damage via regulating P66shc and MnSOD. Cell Physiol Biochem 2015;37:1240-1256.

8 Pieczenik SR, Neustadt J: Mitochondrial dysfunction and molecular pathways of disease. Exp Mol Pathol 2007;83:84-92.

-9 Ding W, Guo H, Xu C, Wang B, Zhang M, Ding F: Mitochondrial reactive oxygen species-mediated NLRP3 inflammasome activation contributes to aldosterone-induced renal tubular cells injury. Oncotarget 2016; 14:17479-17491.

10 Ding W, Xu C, Wang B, Zhang M: Rotenone Attenuates Renal Injury in Aldosterone-Infused Rats by Inhibiting Oxidative Stress, Mitochondrial Dysfunction, and Inflammasome Activation. Med Sci Monit 2015;21:3136-3143.

11 Shen J, Wang L, Jiang N, Mou S, Zhang M, Gu L, Shao X, Wang Q, Qi C, Li S, Wang W, Che X, Ni Z: NLRP3 


\section{Cellular Physiology Cell Physiol Biochem 2017;44:741-750

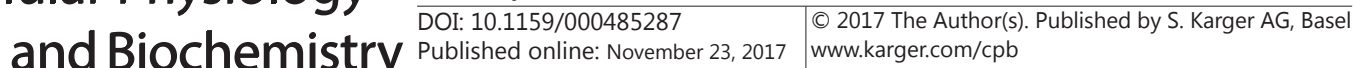

inflammasome mediates contrast media-induced acute kidney injury by regulating cell apoptosis. Sci Rep 2016; 6:34682

12 Wen Y, Liu Y, Tang T, Lv L, Liu H, Ma K, Liu B: NLRP3 inflammasome activation is involved in Ang II-induced kidney damage via mitochondrial dysfunction. Oncotarget 2016;7:54290-54302.

13 Trnka J, Blaikie FH, Smith RA, Murphy MP: A mitochondria-targeted nitroxide is reduced to its hydroxylamine by ubiquinol in mitochondria. Free Radic Biol Med 2008;44:1406-1419.

14 Pan S, Wang N, Bisetto S, Yi B, Sheu SS: Downregulation of adenine nucleotide translocator 1 exacerbates tumor necrosis factor- $\alpha$-mediated cardiac inflammatory responses. Am J Physiol Heart Circ Physiol 2015;308:H39-48.

15 Patil NK, Parajuli N, MacMillan-Crow LA, Mayeux PR: Inactivation of renal mitochondrial respiratory complexes and manganese superoxide dismutase during sepsis: mitochondria-targeted antioxidant mitigates injury. Am J Physiol Renal Physiol 2014;306:F734-743.

16 Guo H, Li H, Ling L, Gu Y, Ding W: Endoplasmic Reticulum Chaperon Tauroursodeoxycholic Acid Attenuates Aldosterone-Infused Renal Injury. Mediators Inflamm 2016;2016:4387031.

17 Guo H, Bi X, Zhou P, Zhu S, Ding W: Nlrp3 deficiency attenuates renal fibrosis and ameliorates mitochondrial dysfunction in a mouse unilateral ureteral obstruction model of chronic kidney disease. Mediators Inflamm 2017;2017:8316560.

18 Xu C, Ding W, Yang L, Yang M, Zhang M, Gu Y: Contributions of endoplasmic reticulum stress and reactive oxygen species to renal injury in aldosterone/salt-induced rats. Nephron Exp Nephrol 2014;1:25-32.

19 Ding W, Yang L, Zhang M, Gu Y: Reactive oxygen species-mediated endoplasmic reticulum stress contributes to aldosterone-induced apoptosis in tubular epithelial cells. Biochem Biophys Res Commun 2012;418:451456.

20 Li X, Wang X, Zheng M, Luan QX: Mitochondrial reactive oxygen species mediate the lipopolysaccharideinduced pro-inflammatory response in human gingival fibroblasts. Exp Cell Res 2016;347:212-221.

-21 Diokmetzidou A, Soumaka E, Kloukina I, Tsikitis M, Makridakis M, Varela A, Davos CH, Georgopoulos S, Anesti V, Vlahou A, Capetanaki Y: Desmin and $\alpha \mathrm{B}$-crystallin interplay in the maintenance of mitochondrial homeostasis and cardiomyocyte survival. J Cell Sci 2016;129:3705-3720.

-22 Ishimoto Y, Inagi R: Mitochondria: a therapeutic target in acute kidney injury. Nephrol Dial Transplant 2016;31:1062-1069.

-23 Güçer S, Talim B, Aşan E, Korkusuz P, Ozen S, Unal S, Kalkanoğlu SH, Kale G, Cağlar M: Focal segmental glomerulosclerosis associated with mitochondrial cytopathy: report of two cases with special emphasis on podocytes. Pediatr Dev Pathol 2005;8:710-717.

24 Su M, Dhoopun AR, Yuan Y, Huang S, Zhu C, Ding G, Liu B, Yang T, Zhang A: Mitochondrial dysfunction is an early event in aldosterone-induced podocyte injury. Am J Physiol Renal Physiol 2013;305:F520-531.

25 Homsi E, Janino P, de Faria JB. Role of caspases on cell death, inflammation, and cell cycle in glycerolinduced acute renal failure. Kidney Int 2006;69:1385-1392.

-26 Vilaysane A, Chun J, Seamone ME, Wang W, Chin R, Hirota S, Li Y, Clark SA, Tschopp J, Trpkov K, Hemmelgarn BR, Beck PL, Muruve DA: The NLRP3 inflammasome promotes renal inflammation and contributes to CKD. J Am Soc Nephrol 2010;21:1732-1744.

-27 Ludwig-Portugall I, Bartok E, Dhana E, Evers BD, Primiano MJ, Hall JP, Franklin BS, Knolle PA, Hornung V, Hartmann G, Boor P, Latz E, Kurts C: An NLRP3-specific inflammasome inhibitor attenuates crystal-induced kidney fibrosis in mice. Kidney Int 2016;90:525-539.

-28 Tse G, Yan BP, Chan YW, Tian XY, Huang Y: Reactive Oxygen Species, Endoplasmic Reticulum Stress and Mitochondrial Dysfunction: The Link with Cardiac Arrhythmogenesis. Front Physiol 2016;7:313.

-29 Safiedeen Z, Andriantsitohaina R, Martinez MC: Dialogue between endoplasmic reticulum and mitochondria as a key actor of vascular dysfunction associated to metabolic disorders. Int J Biochem Cell Biol 2016;77:10-14.

30 Ding W, Wang B, Zhang M, Gu Y:Involvement of Endoplasmic Reticulum Stress in Uremic Cardiomyopathy: Protective Effects of Tauroursodeoxycholic Acid. Cell Physiol Biochem 2016;38:141-152.

31 Wang J, Yang X, Zhang J: Bridges between mitochondrial oxidative stress, ER stress and mTOR signaling in pancreatic $\beta$ cells. Cell Signal 2016;28:1099-1104.

-32 Nakahira K, Haspel JA, Rathinam VA, Lee SJ, Dolinay T, Lam HC, Englert JA, Rabinovitch M, Cernadas M, Kim HP, Fitzgerald KA, Ryter SW, Choi AM: Autophagy proteins regulate innate immune responses by inhibiting the release mitochondrial DNA mediated by the NALP3 inflammasome. Nat Immunol 2011;12:222-230. 